\title{
ESTABILIDADE E ADAPTABILIDADE DE HÍBRIDOS DUPLOS EXPERIMENTAIS DE MILHO
}

\section{STABILITY AND ADAPTABILITY OF EXPERIMENTAL DOUBLE HYBRIDS IN CORN}

\author{
Ricardo Machado da SILVA ${ }^{1}$ \\ Leonardo Novaes ROSSE ${ }^{2}$ \\ José Roberto MÔRO ${ }^{3}$
}

\begin{abstract}
RESUMO
A interação de genótipos com ambientes é um dos principais complicadores dos programas de melhoramento genético, pois dificulta a etapa de avaliação e recomendação de materiais genéticos de boa estabilidade fenotípica. Procurou-se nesse trabalho, estudar a estabilidade fenotípica de híbridos duplos experimentais de milho por meio do modelo dos efeitos principais aditivos e interação multiplicativa (AMMI). Avaliaram-se para esse objetivo 124 híbridos duplos experimentais mais cinco híbridos duplos comerciais como testemunhas, divididos em quatro grupos de ensaios delineados em látice $6 \times 6$ com três repetições e instalados em três ambientes distintos do estado de São Paulo: JAB-1 e JAB-2 em Jaboticabal nos meses de dezembro de 1995 e janeiro de 1996, respectivamente, e BAR-1 em Barretos em dezembro de 1995. Constatouse que o modelo em estudo mostrou diferenças significativas no comportamento dos materiais genéticos, mesmo empregando pequeno número de ambientes. Esses ambientes foram bastante contrastantes e contribuíram de maneira preponderante para a significância dos escores. Numa situação em que se tem restrições quanto ao número de ambientes para se inferir sobre a estabilidade de materiais genéticos, o emprego da metodologia AMMI mostra-se bastante adequada.
\end{abstract}

Palavras-chave: AMMI, biplot, Zea mays.

\begin{abstract}
The interaction genotype-environment represent one of the most important complications in genetic improvement programs, difficulting the evaluation and advise of genetics materials with good phenotypical stability. The objective of this work was to study the phenotypical stability of double experimental corn hybrids by means of the Additive Main Effects and Multiplicative Interaction Analysis (AMMI) models. For this purpose 124 double experimental hybrids were evaluated plus 5 double commercial hybrids as controls. These hybrids were divided in 4 essay groups $(6 \times 6)$ with 3 repeats and installed in three different environments of the State of São Paulo (Brazil): JAB-1 and JAB-2 in Jaboticabal, during the months of December in 1995 and January in 1996, respectively, and BAR-1 in Barretos in December, 1995. We were able to conclude that the model was usefull for to detect significant differences in the behaviour of the genetics materials, even using a small number of environments. These environments were, quite different, and contributed a lot for the significance of the scores. In a situation where there are only few environments, the AMMI model seems to be very adequate for this kind of studies.
\end{abstract}

Key-words: AMMI, biplot, Zea mays.

\footnotetext{
${ }^{1}$ Professor do Depto de Ciências Agrárias/Universidade de Taubaté/SP.

${ }^{2}$ Veracel Celulose S.A. Rua Davi J. Fadini, 300 Eunápolis/.BA Caixa Postal: 21 45.823-035. E-mail: leonardo.rosse@veracel.com.br (autor correspondente).

${ }^{3}$ Professor do Depto de Biologia Aplicada à Agropecuária - FCAV/UNESP - Jaboticabal/SP.
} 
SILVA, RM. et al. Estabilidade e adaptabilidade de híbridos...

\section{INTRODUÇÃO}

Dentre os vegetais, o milho é uma das espécies mais estudada no campo da genética, o que resultou em significativa contribuição para o melhoramento de suas características de interesse agronômico. Apesar da importância dessas características, constata-se que o parâmetro norteador na escolha de um cultivar de milho tem sido a produtividade de grãos.

$\mathrm{Na}$ prática, verifica-se acentuada variação na produtividade de grãos do milho entre locais e/ou ao longo dos anos, especialmente devido a fatores de natureza ambiental. Essa variação no comportamento produtivo de cultivares em diferentes ambientes é muito comum e recebe a denominação de interação de materiais genéticos com ambientes (4).

Pela importância do fenômeno da interação, cabe ao melhorista avaliar sua magnitude $e$ significância, quantificar seus efeitos sobre as técnicas de melhoramento e estratégias de difusão de tecnologia e, fornecer subsídios que possibilitem adotar procedimentos para sua minimização e/ou seu aproveitamento (3).

No estudo da interação de genótipos $x$ ambientes, várias metodologias foram propostas com o objetivo de atenuá-la. Destaca-se àquelas que se baseiam em regressão linear e, dentre elas, as propostas por Eberhart e Russell (5) e Cruz et al. (2), que são respectivamente, uni e bissegmentadas, como as mais utilizadas.

Recentemente, tem-se empregado técnicas multivariadas para avaliar o comportamento dos genótipos frente aos vários locais $(10,7,9)$. Dentre essas técnicas, o modelo dos efeitos principais aditivos e interação multiplicativa (AMMI) tem sido bastante empregado em várias culturas e em vários países. No Brasil, são poucos os relatos em que essa técnica foi utilizada. Destaca-se os estudos de Pereira e Costa (6) que analisaram a estabilidade de produção de materiais genéticos de batata no Rio Grande do Sul. Outro estudo objetivou avaliar a estabilidade e adaptabilidade de cultivares de milho no estado do Mato
Grosso do Sul empregando o modelo AMMI e várias outras metodologias (1). Esse autor verificou pelo AMMI, que os três primeiros componentes principais foram significativos e captaram $71 \%$ da variação devida à interação. Porém, salienta que o primeiro componente explicou apenas $28,6 \%$ de toda a variação. Desse modo, a interpretação dos resultados ficou prejudicada devido a necessidade de haver mais de um componente para explicar a variação dessa interação.

Pelo exposto, o objetivo do presente trabalho foi de avaliar as interações de materiais genéticos com ambientes em ensaios de híbridos duplos experimentais de milho visando identificar os materiais mais estáveis e promissores em cultivos tardios ou de safrinha utilizando a metodologia AMMI.

\section{MATERIAL E MÉTODOS}

Avaliaram-se 124 híbridos duplos experimentais de milho e cinco híbridos duplos comerciais como testemunhas, sendo eles: BR-201 da Embrapa (T1) e os híbridos duplos XL-678 (T2), XL-660 (T3), XL-604 (T4) e XL-655 (T5) da empresa Braskalb Agropecuária Brasileira Ltda, atualmente Dekalb.

Três experimentos foram implantados em áreas distintas, obedecendo a seguinte distribuição: Experimento JAB-1 em Jaboticabal-SP em dezembro de 1995, Experimento BAR-1 em Barretos-SP em dezembro de 1995 e Experimento JAB-2 em Jaboticabal-SP em janeiro de 1996, empregando-se o delineamento em látice com três repetições. Devido ao grande número de materiais, cada experimento foi dividido em quatro grupos de ensaios $6 \times 6$ com três repetições, como apresentado na Tabela 1.

Avaliaram-se a produtividade de grãos $\left(\mathrm{kg} \mathrm{ha}^{-1}\right)$ corrigido para $13 \%$ de umidade em parcelas constituídas de duas linhas de cinco metros, espaçadas $0,20 \mathrm{~m}$ entre plantas e $0,90 \mathrm{~m}$ entre linhas. Aárea útil da parcela foi de nove $\mathrm{m}^{2} \mathrm{com} 50$ plantas.

TABELA 1 - Relação dos materiais genéticos e testemunhas avaliadas e suas disposições nos grupos de ensaio nos três locais de experimentação. Jaboticabal, 1997.

\begin{tabular}{|c|c|c|c|c|}
\hline \multirow{2}{*}{ Locais } & \multicolumn{4}{|c|}{ Grupos de Ensaios } \\
\hline & 1 & 2 & 3 & 4 \\
\hline \multirow{4}{*}{ JAB-1 } & 1 a 31 & 32 a 62 & 63 a 93 & 94 a 124 \\
\hline & + & + & + & + \\
\hline & $\mathrm{T} 1, \mathrm{~T} 2, \mathrm{~T} 3, \mathrm{~T} 4, \mathrm{~T} 5$ & $\mathrm{~T} 1, \mathrm{~T} 2, \mathrm{~T} 3, \mathrm{~T} 4, \mathrm{~T} 5$ & $\mathrm{~T} 1, \mathrm{~T} 2, \mathrm{~T} 3, \mathrm{~T} 4, \mathrm{~T} 5$ & $\mathrm{~T} 1, \mathrm{~T} 2, \mathrm{~T} 3, \mathrm{~T} 4, \mathrm{~T} 5$ \\
\hline & 5 & 6 & 7 & 8 \\
\hline \multirow{4}{*}{ JAB-2 } & 1 a 31 & 32 a 62 & 63 a 93 & 94 a 124 \\
\hline & + & + & + & + \\
\hline & $\mathrm{T} 1, \mathrm{~T} 2, \mathrm{~T} 3, \mathrm{~T} 4, \mathrm{~T} 5$ & $\mathrm{~T} 1, \mathrm{~T} 2, \mathrm{~T} 3, \mathrm{~T} 4, \mathrm{~T} 5$ & $\mathrm{~T} 1, \mathrm{~T} 2, \mathrm{~T} 3, \mathrm{~T} 4, \mathrm{~T} 5$ & $\mathrm{~T} 1, \mathrm{~T} 2, \mathrm{~T} 3, \mathrm{~T} 4, \mathrm{~T} 5$ \\
\hline & 9 & 10 & 11 & 12 \\
\hline \multirow{3}{*}{ BAR-1 } & 1 a 31 & 32 a 62 & 63 a 93 & 94 a 124 \\
\hline & + & + & + & + \\
\hline & $\mathrm{T} 1, \mathrm{~T} 2, \mathrm{~T} 3, \mathrm{~T} 4, \mathrm{~T} 5$ & $\mathrm{~T} 1, \mathrm{~T} 2, \mathrm{~T} 3, \mathrm{~T} 4, \mathrm{~T} 5$ & $\mathrm{~T} 1, \mathrm{~T} 2, \mathrm{~T} 3, \mathrm{~T} 4, \mathrm{~T} 5$ & $\mathrm{~T} 1, \mathrm{~T} 2, \mathrm{~T} 3, \mathrm{~T} 4, \mathrm{~T} 5$ \\
\hline
\end{tabular}


Detectado diferença significativa da interação de materiais genéticos com ambientes, procedeu-se a análise da estabilidade e adaptabilidade utilizandose o modelo AMMI. A parte aditiva do modelo foi estimado pelo modo usual da ANOVA e a porção multiplicativa pela análise da decomposição dos valores singulares (DVS) como apresentado por Duarte e Vencovsky (4). O modelo AMMI é assim expresso:

$$
Y_{i j}=\mu+G_{i}+E_{j}+\sum_{n=1}^{N} \lambda_{n} \alpha_{i n} \gamma_{j n}+R_{i j}+e_{i j}
$$

em que:

$Y_{\mathrm{ij}}$ : é a produtividade média observada de cada genótipo i no ambiente j;

$\mu$ : é a média geral;

$\mathrm{G}_{\mathrm{i}}$ : é o efeito fixo do genótipo i;

$E_{j}$ : é o efeito aleatório do ambiente j;

$\lambda_{n}$ : é o n-ésimo valor singular da interação genótipos $\mathrm{x}$ ambientes;

$\alpha_{1 n}$ e $\gamma_{n n}$ : são os elementos relacionados ao genótipo $\mathrm{i}$ e ao ambiente $\mathrm{j}$ dos vetores singulares. $\mathrm{O}$ índice $\mathrm{n}$ tomado até $\mathrm{N}$ no somatório, determina uma aproximação de quadrados mínimos para a matriz de materiais genéticos $x$ ambientes;

$\mathrm{R}_{\mathrm{ij}}$ : é o resíduo;

$\mathrm{e}_{\mathrm{i}: \mathrm{i}}$ é o erro aleatório associado às parcelas.

Para essa análise, foi utilizado o PROC IML/ SAS como apresentado por Duarte e Vencovsky (4).

Através do biplot, realizou-se a análise gráfica com a produtividade na abcissa e os escores dos materiais genéticos e ambientes na ordenada, permitindo com isso, uma melhor visualização do comportamento desses materiais genéticos.

\section{RESULTADOS E DISCUSSÃO}

Os resultados da análise de variância conjunta para cada um dos grupos de ensaios $(1,5,9 ; 2,6$, $10 ; 3,7,11$ e $4,8,12)$ mostraram haver diferenças altamente significativas $(P<0,01)$ para as fontes de variação de materiais genéticos, de ambientes e da interação entre elas (Tabela 2). A significância dessa última fonte de variação é um indicativo de que os materiais genéticos apresentaram comportamento diferenciado nos vários ambientes, sugerindo assim, a realização de uma análise mais criteriosa para identificar quais deles exibem uma menor oscilação nesses ambientes estudados.

Verifica-se que a produtividade média de grãos variou de aproximadamente $4263 \mathrm{~kg} \cdot \mathrm{ha}^{-1}$ para o grupo 4, 8, 12 até $5169 \mathrm{~kg}$ ha $^{-1}$ para o grupo 1, 5, 9 (Tabela 2). Embora essa amplitude de variação não seja tão elevada, é um indicativo de que tanto os materiais genéticos quanto os ambientes responderam de forma diferenciada. Os coeficientes de variação (CV\%) de cada um dos experimentos mostraram-se de baixa magnitude, permitindo inferir que eles foram bem conduzidos.

TABELA2 - Resumo da análise de variância conjunta dos ensaios realizados em Jaboticabal e Barretos para o caráter produtividade de grãos dos materiais genéticos de milho no ano agrícola de 1995/96. Jaboticabal, 1997.

\begin{tabular}{|c|c|c|c|c|c|}
\hline \multirow{2}{*}{ FV } & \multirow{2}{*}{ GL } & \multicolumn{4}{|c|}{ QM } \\
\hline & & $(1,5,9)$ & $(2,6,10)$ & $(3,7,11)$ & $(4,8,12)$ \\
\hline $\begin{array}{l}\text { Materiais genéticos } \\
(\mathrm{G})\end{array}$ & 35 & $1146380,36^{* *}$ & $798128,78^{* *}$ & $748092,30^{* *}$ & $706572,85^{\star *}$ \\
\hline $\begin{array}{c}\operatorname{Amb}(A) \\
G X A\end{array}$ & $\begin{array}{c}2 \\
70\end{array}$ & $\begin{array}{r}211625743,01^{* *} \\
232667,66^{* *}\end{array}$ & $\begin{array}{r}254333544,57^{* *} \\
255141,12^{* *}\end{array}$ & $\begin{array}{r}157550738,95^{* *} \\
182459,04^{* *}\end{array}$ & $\begin{array}{r}146810140,13^{* *} \\
411878,38^{* *}\end{array}$ \\
\hline PC1 & 36 & $326699,59^{* *}$ & $349404,69^{* *}$ & $199131,83^{*}$ & $512789,25^{* *}$ \\
\hline PC2 & 34 & $133104,44^{\text {ns }}$ & $155332,62^{\text {ns }}$ & $164805,49^{\text {ns }}$ & $305031,58^{*}$ \\
\hline Erro Médio & 210 & 124223,06 & 166201,51 & 129996,35 & 189596,46 \\
\hline $\begin{array}{l}\text { Média Geral } \\
\text { CV }(\%)\end{array}$ & & $\begin{array}{c}5169,9 \\
6,8\end{array}$ & $\begin{array}{c}4729,7 \\
8,6\end{array}$ & $\begin{array}{c}4438,0 \\
8,1\end{array}$ & $\begin{array}{c}4263,6 \\
10,2\end{array}$ \\
\hline
\end{tabular}

**, * : Significativo a $1 \%$ e $5 \%$ de probabilidade pelo teste $\mathrm{F}$, respectivamente.

ns: não significativo.

Procedendo à decomposição da interação materiais genéticos $x$ ambientes de cada um dos experimentos através do modelo AMMI (Tabela 2), constatou-se que em todos os grupos de ensaios o primeiro componente principal (PC1) foi altamente significativo $(P<0,01)$. Já o segundo componente $(P C 2)$, foi significativo somente para o ensaio 4, 8, $12(P<0,05)$. De um modo geral, o primeiro componente explicou mais de $50 \%$ da variação total da interação (TABELA 3 ), evidenciando que o mesmo conseguiu mostrar a maior parte da variação contida nesse conjunto de materiais genéticos. Essa quantidade foi superior à 
SILVA, RM. et al. Estabilidade e adaptabilidade de híbridos...

encontrada por Arias (1), embora esse autor tenha considerado um número maior de ambientes, fato não ocorrido no presente estudo. De um certo modo, a não significância do segundo componente já era esperado, devido ao baixo número de ambientes considerados nessa pesquisa.

TABELA 3 - Proporção da soma de quadrados da interação de materiais genéticos de milho $\mathrm{x}$ ambientes explicada por cada eixo singular. Jaboticabal, 1997.

\begin{tabular}{ccccccccc}
\hline \multirow{2}{*}{$\begin{array}{c}\text { Eixo } \\
\text { Singular }\end{array}$} & \multicolumn{3}{c}{ Proporção (SQGXE/Eixo) } & \multicolumn{4}{c}{ Proporção acumulada (\%) } \\
\cline { 2 - 8 } & $(1,5,9)$ & $(2,6,10)$ & $(3,7,11)$ & $(4,8,12)$ & $(1,5,9)$ & $(2,6,10)$ & $(3,7,11)$ & $(4,8,12)$ \\
\hline 1 & 0,722 & 0,704 & 0,562 & 0,641 & 72,2 & 70,4 & 56,2 & 64,1 \\
2 & 0,278 & 0,296 & 0,438 & 0,359 & 100 & 100 & 100 & 100 \\
\hline
\end{tabular}

A significância dos componentes principais está relacionada com as magnitudes dos escores para materiais genéticos e para ambientes, explicando assim, parte da variação da interação. Pelos valores encontrados, pode-se inferir que há padrões de interação diferenciado de materiais genéticos e ambientes, ou seja, de estabilidade genotípica e ambiental. A estabilidade dos materiais genéticos dos vários grupos de ensaios pode ser identificada pela variabilidade na produtividade de grãos e pelos valores dos escores do primeiro componente principal e, no caso do ensaio $4,8,12$, também pelo segundo componente.

Escores altos não importando se positivos ou negativos, indicam grandes interações, ou seja, baixa estabilidade. Por outro lado, escores próximos a zero, refletem baixas interações, acarretando consequentemente, alta estabilidade. Nesse sentido, constatou-se que a amplitude de variação dos escores foi alta para todos os grupos de ensaios, atribuindo aos ambientes os seus maiores valores. Esses altos valores para ambientes indicam que eles apresentaram níveis de produtividades bem distintos. $O$ ambiente BAR-1 foi o que apresentou a menor produtividade devido a forte seca durante o período vegetativo do milho.

Para ilustrar bem toda essa variação, as Figuras de 1 a 4 foram elaboradas, considerando apenas alguns materiais genéticos e todos os três ambientes. Elas facilitam a identificação de quais materiais genéticos e/ou ambientes contribuíram mais para a interação, informando também sobre a estabilidade de materiais genéticos e de ambientes. Assim, um genótipo com características desejáveis é aquele que contempla escores próximo a zero, e alta produtividade média. Nesse sentido, observa-se que os materiais genéticos mais promissores do ensaio $1,5,9$ foram o 1, 3, 5, 16 e a testemunha T1 (Figura1). Desse conjunto, o 1 foi o de maior destaque com produti- vidade acima de $6000 \mathrm{~kg} \cdot \mathrm{ha}^{-1}$.

Os materiais genéticos 44, 57 e 59 e a testemunha T1 do grupo de ensaio $2,6,10$, apresentaram escores próximos a zero, significando baixas interações (Figura 2). As suas produtividades foram semelhantes entre si e acima da média geral (4730 kg.ha-1) o que é desejável. Por outro lado, o material genético 32 apresentou alta produtividade, porém baixa estabilidade, não se enquadrando dentro das características desejáveis de um bom material genético pelo método AMMI.

Os materiais genéticos mais estáveis e que associaram produtividades acima da média geral para o ensaio $3,7,11$ foram o $66,75,82,89$ e as testemunhas T1, T2 e T3 (Figura 3) e, para o ensaio 4, 8, 12, os materiais genéticos 95, 100, 107 e a testemunha T2 (Figura 4).

Em função do segundo componente principal ter sido também significativo para o grupo de ensaio 4,8 e 12, procedeu-se à sua representação gráfica considerando na abcissa o primeiro componente principal e na ordenada o segundo (Figura 5). Para facilitar a visualização dessa Figura, construiu-se um círculo onde o seu centro é a origem dos eixos. Por essa representação, os materiais genéticos e os ambientes mais estáveis são aqueles que se encontram dentro desse círculo e cujo os pontos situam-se preferencialmente próximo à origem. Destaca-se entre eles o material 107 como sendo o mais estável, além também dos de número 95, 96, 101, 111, 121 e as testemunhas T1 e T2. Quanto aos ambientes, nota-se que seus escores foram altos, situando-os em pontos distantes da origem, refletindo assim, em forte contribuição para a interação, ou seja, baixa estabilidade ambiental. É possível inferir também, que a testemunha T5 mostrou-se de adaptação específica para o ambiente BAR-1, o mesmo podendo mencionar para a T3 no JAB-2. 
SILVA, RM. et al. Estabilidade e adaptabilidade de híbridos...

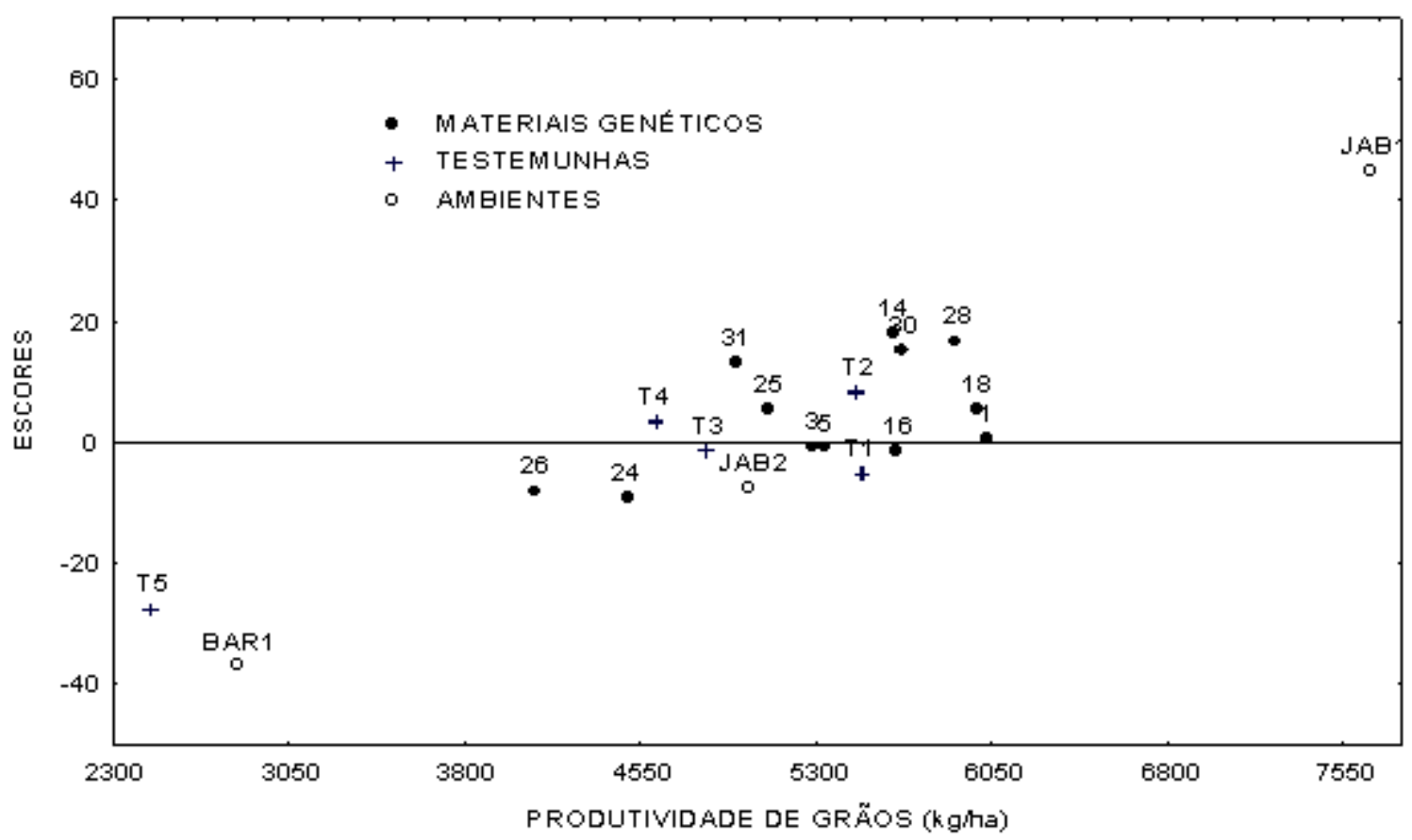

FIGURA 1 - Gráfico referente a análise de estabilidade e produtividade de grãos dos materiais genéticos de milho nos Ensaios 1, 5 e 9. Jaboticabal, 1997.

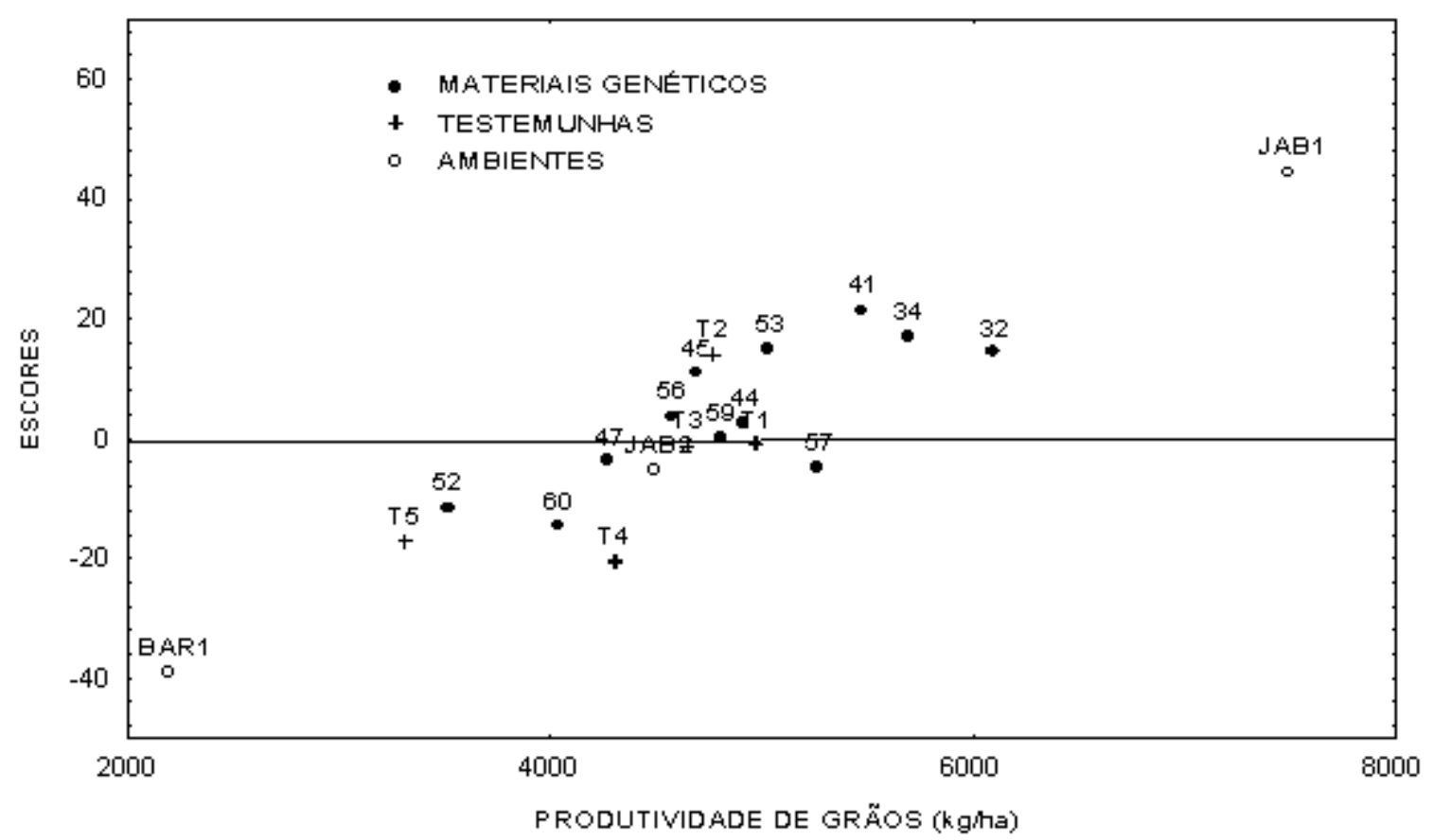

FIGURA 2 - Gráfico referente a análise de estabilidade e produtividade de grãos dos materiais genéticos de milho nos Ensaios 2, 6 e 10. Jaboticabal, 1997. 
SILVA, RM. et al. Estabilidade e adaptabilidade de híbridos...

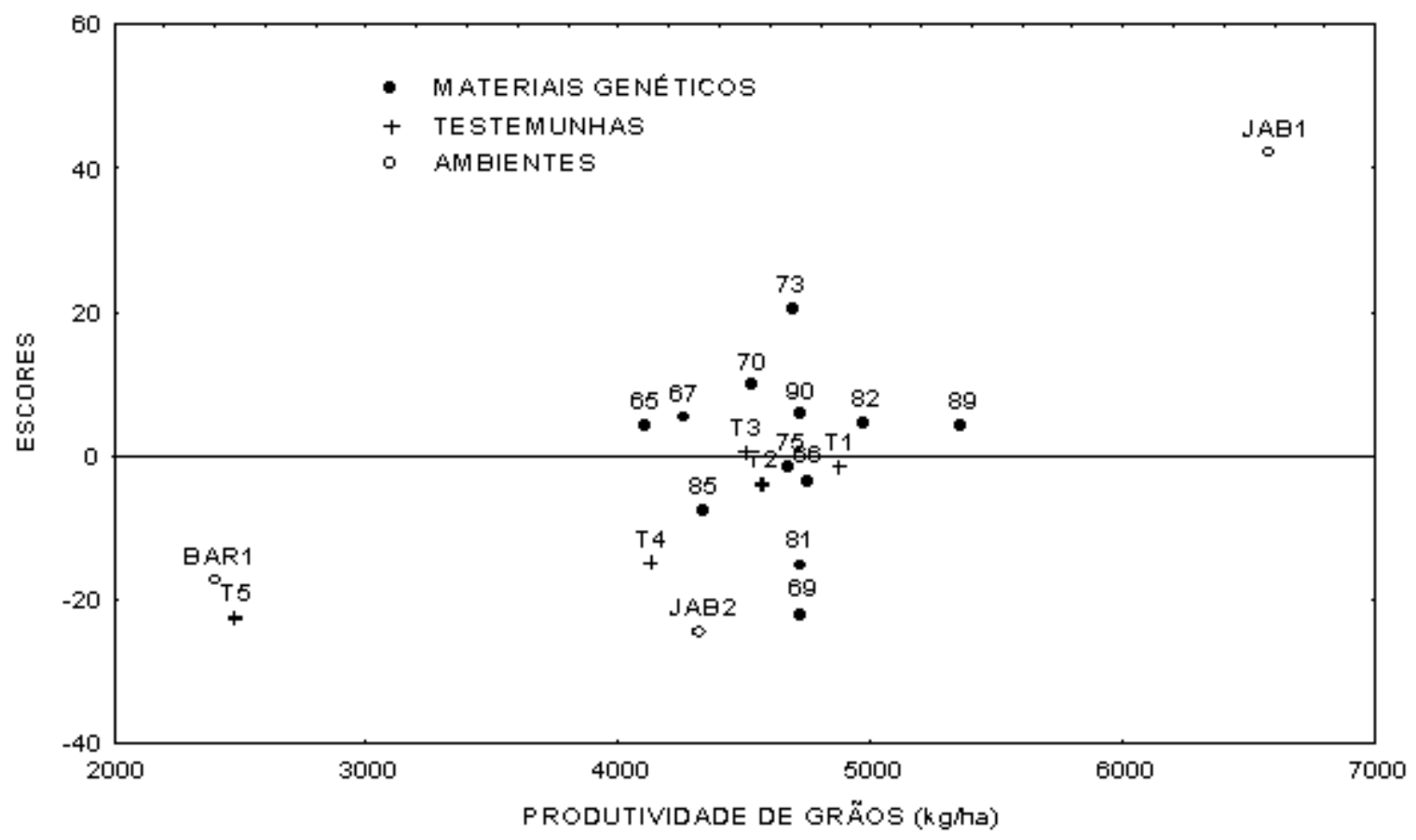

FIGURA 3 - Gráfico referente a análise de estabilidade e produtividade de grãos dos materiais genéticos de milho nos Ensaios 3, 7 e 11. Jaboticabal, 1997.

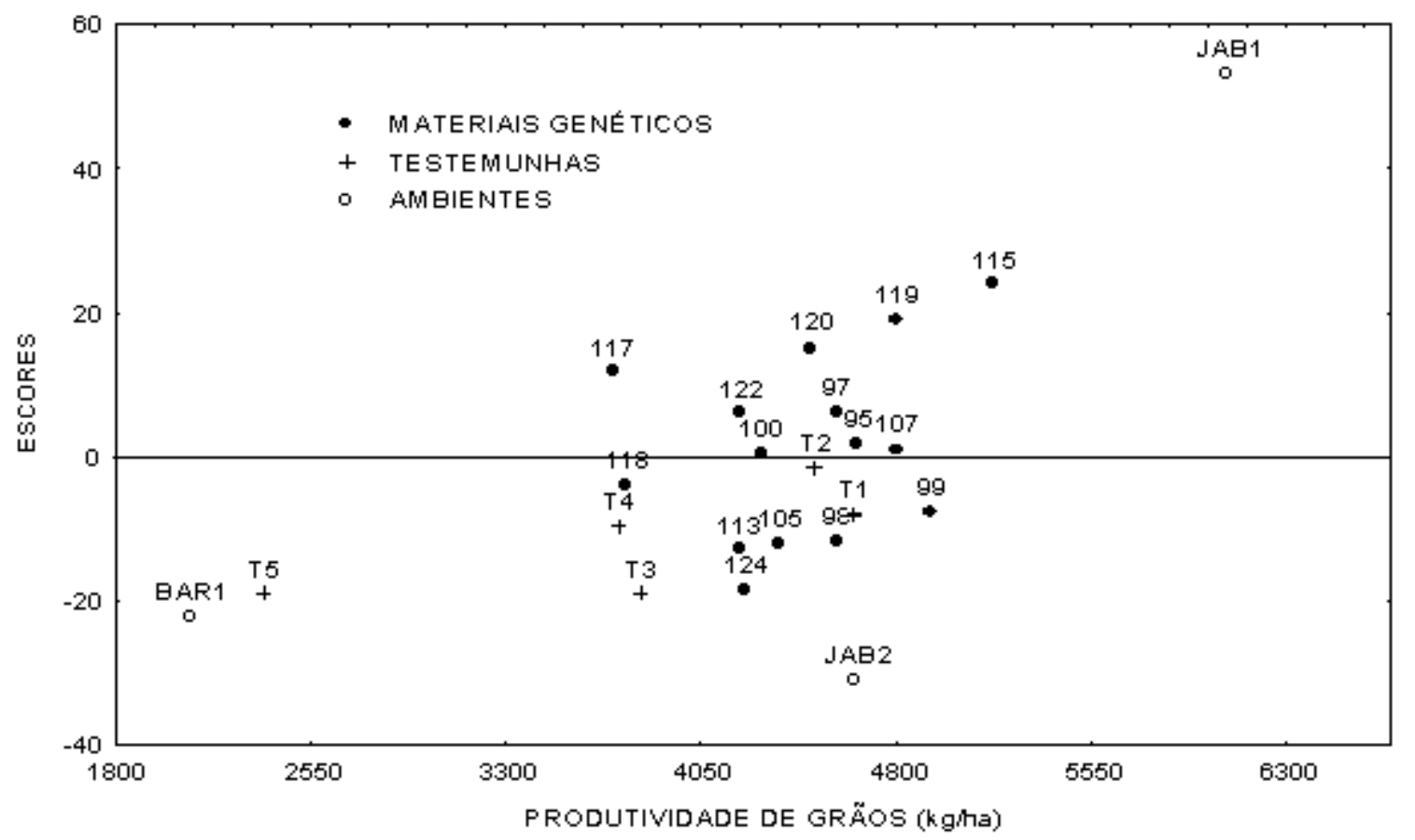

FIGURA 4 - Gráfico referente a análise de estabilidade e produtividade de grãos dos materiais genéticos de milho nos Ensaios 4, 8 e 12. Jaboticabal, 1997. 
Avaliando-se os mesmos materiais para estabilidade e adaptabilidade utilizando-se o método de regressão linear proposto por Eberhart e Russell (5), Silva (8) verificou-se que todos eles mostraram-se estáveis e de boa previsibilidade. No entanto, isso ocorreu devido as linhas de regressão associadas a cada material terem sido testadas com apenas um grau de liberdade. Empregando o método AMMI, foi possível discriminar melhor o comportamento de cada um desses materiais genéticos, observando o seu desempenho em cada um dos ambientes.

Comparando esses resultados com os obtidos por Silva (8), constatou-se, por exemplo, que os ma- teriais genéticos 14,28 e 30 do grupo de ensaio 1,5 e 9; 32, 34 e 41 do grupo de ensaio 2, 6, 10; 69, 73 e T4 do grupo de ensaio 3, 7, 11 e 115, 119 e T3 do grupo 4, 8, 12 considerados estáveis pelo método da regressão apresentaram-se não muito estáveis pelo método AMMI.

Os resultados apresentados pela metodologia AMMI em relação aos de regressão linear, possibilita inferir que em situações onde se tem restrições quanto ao número de ambientes, o emprego da metodologia AMMI mostra-se mais adequada em discriminar melhor o comportamento dos materiais genéticos avaliados.

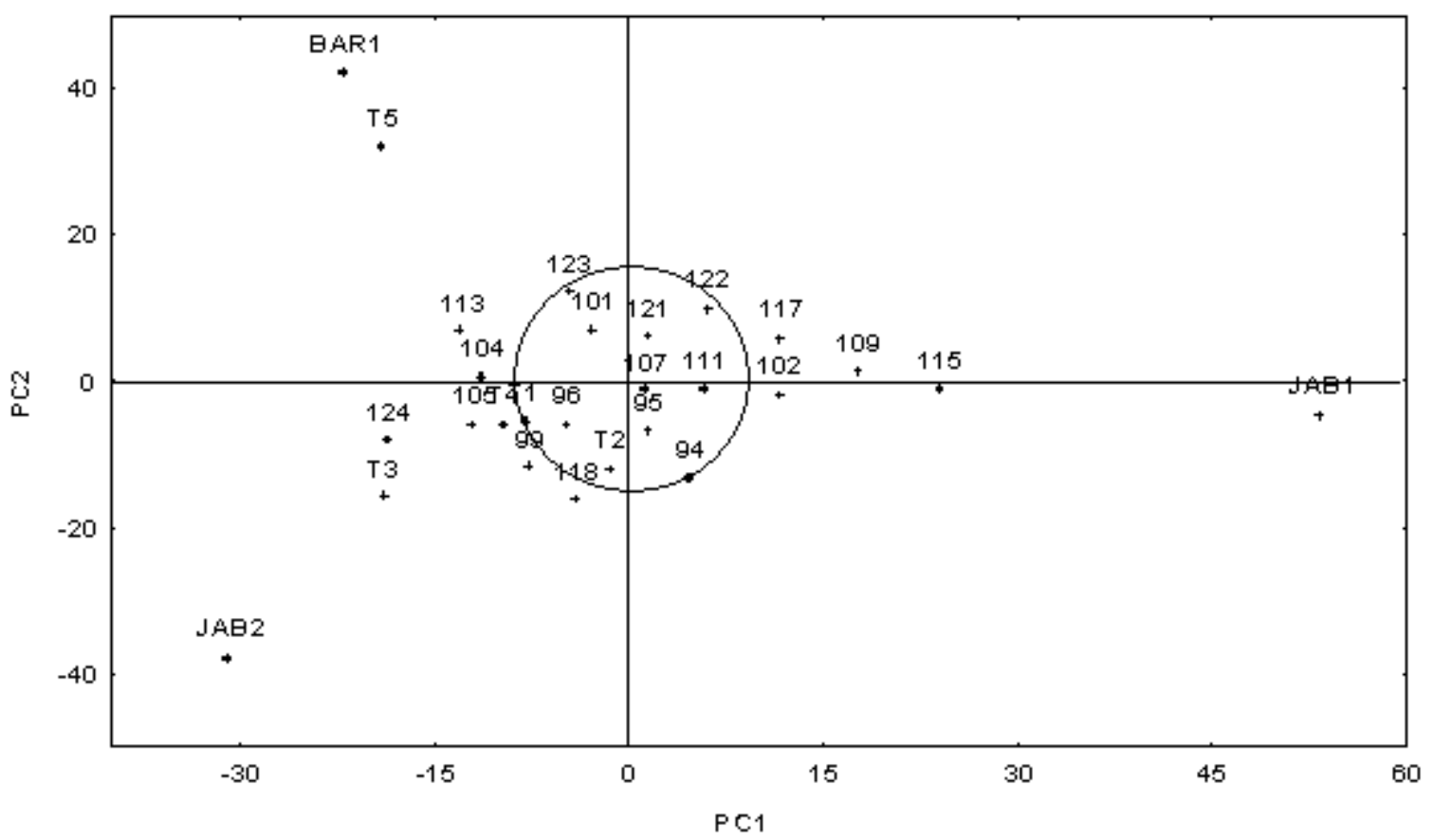

FIGURA 5 - Gráfico referente a análise de estabilidade e produtividade de grãos dos materiais genéticos de milho nos Ensaios 4, 8 e 12 considerando os dois componentes principais. Jaboticabal, 1997.

\section{CONCLUSÕES}

A metodologia AMMI permite diferenciar o comportamento dos materiais genéticos dos diferentes grupos de ensaios e, também dos ambientes quanto à estabilidade de produtividade de grãos.

Os ambientes, de um modo geral, mostram produtividade de grãos bastante divergentes entre si.

As testemunhas T4 e T5 apresentam, no geral, menores produtividade de grãos em relação às demais. 
SILVA, RM. et al. Estabilidade e adaptabilidade de híbridos...

\section{REFERÊNCIAS BIBLIOGRÁFICAS}

1 ARIAS, E.R.A. Adaptabilidade e estabilidade das cultivares de milho avaliadas no estado de Mato Grosso do Sul e avanço genético obtido no período de 1986/87 a 1993/94. Lavras, 1996. 118 f. Tese (Doutorado em Genética e Melhoramento de Plantas) - Universidade Federal de Lavras.

2 CRUZ, C.D.; TORRES, R.A.; VENCOVSKY, R. An alternative approach to the stability analysis proposed by Silva and Barreto. Revista Brasileira de Genética, Ribeirão Preto, v.12, n.3, p.567-580, 1989.

3 CRUZ, C.D., REGAZZI, A.J. Modelos biométricos aplicados ao melhoramento genético. Viçosa: UFV, 1994, 390p.

4 DUARTE, J.B., VENCOVSKY, R. Interação materiais genéticos $\mathbf{x}$ ambientes: uma introdução à análise "AMMl”. Ribeirão Preto: Sociedade Brasileira de Genética, 1999.60 p. (Série Monografia, n.9)

5 EBERHART, S.A., RUSSELL, W.A. Stability parameters for comparing varieties. Crop Science, Madison, v.6, n.1, p.36-40, 1966.

6 PEREIRA, A.S., COSTA, D.M. Análise de estabilidade de produção de materiais genéticos de batata no Rio Grande do Sul. Pesquisa Agropecuária Brasileira, Brasília, v.33, n.4, p.405-409, 1998.

7 SILVA, J.G.C. Agrupamento de materiais genéticos segundo a adaptabilidade ao ambiente. Pesquisa Agropecuária Brasileira, Brasília, v.30, n.9, p.1141-1154, 1995.

8 SILVA, R.M. Produtividade, estabilidade e adaptabilidade de híbridos duplos experimentais de milho (Zea mays L.). Jaboticabal, 1997. 75f. Dissertação (Mestrado em Genética e Melhoramento de Plantas) - Faculdade de Ciências Agrárias e Veterinária - Universidade Estadual Paulista Júlio de Mesquita Filho.

9 VAN EUWIJK, F.A. Linear and bilinear models for the analysis of multi-environments trials: I. An inventory of models. Euphytica, Dordrecht, v.84, p.1-7, 1995

10 ZOBEL,R.W., WRIGHT, A.J., GAUCH, H.G. Statistical analysis of a yield trial. Agronomy Journal, Madison, v.80, p.388-393, 1988. 